\title{
NOTICIARIO
}

\section{NUEVAS AVES FÓSILES \\ DEL YACIMIENTO \\ MESOPLEISTOCÉNICO \\ DE AMBRONA (SORIA, ESPAÑA)}

\author{
NEWS FOSSIL BIRDS FROM THE MIDDLE \\ PLEISTOCENE SITE OF AMBRONA \\ (SORIA, SPAIN)
}

ANTONIO SÁNCHEZ MARCO (*)

\section{RESUMEN}

Se da cuenta de seis nuevos restos fósiles avianos procedentes del yacimiento del Pleistoceno medio de Ambrona y se ofrece una lista revisada de los taxones identificados hasta ahora en Torralba y Ambrona. También, a partir de los taxones que se han reconocido hasta ahora, se hace alguna indicación sobre las condiciones paleoecológicas de la región.

\section{ABSTRACT \\ Taxonomic identification of six new fossill bird bones from the Middle Pleistocene site at Ambrona is presented along with a complete and revised list of the bird remains from the contemporary sites of Torralba and Ambrona. Given the species present, a reconstruction of the regional paleoecology is proposed.}

Palabras clave: Aves fósiles. Pleistoceno medio. Ambrona. España.

Key words: Bird bones. Middle Pleistocene. Ambrona. Spain.

(*) Departamento de Paleobiología. Museo Nacional de Ciencias Naturales. José Gutiérrez Abascal, 2. 28006 Madrid.

El artículo fue remitido en su versión final el 22-I-99.

\section{INTRODUCCIÓN}

El yacimiento de Ambrona recibe su nombre del pueblo soriano en cuyo término municipal se encuentra (Fig. 1). Se halla en la vertiente izquierda del valle del río Masegar, a una altura poco superior a 1100 m.s.n.m. Existe en este yacimiento un conjunto lítico de factura Achelense (Howell, 1962; Biberson, 1964). Las asociaciones, tanto de macro como de micromamíferos, se corresponden con las del Pleistoceno medio (Pérez et alii, 1997).

Los nuevos fósiles de aves se encontraron entre el material que extrajo C. Sesé deAmbrona para realizar su estudio de los micromamíferos de este yacimiento (Sesé, 1986). Las muestras fueron toma-

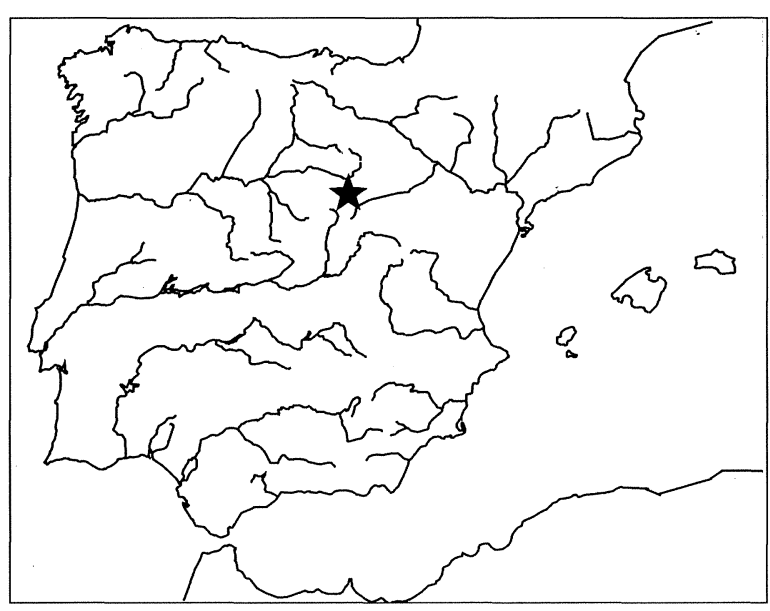

Fig. 1. Situación geográfica de Ambrona (Soria). 
das durante las campañas que dirigieron F.C. Howe11 y L.G. Freeman entre los años 1980 y 1983. Todas proceden del Complejo Inferior deAmbrona; en particular, de las margas que se encuentran por encima de los niveles llamados "pavimentos" fluviales. Por tanto, las unidades de procedencia serían las comprendidas entre AS3 y AS6, formadas por materiales depositados en ambiente fluvio-lacustre (Pérez et alii, e.p.).

\section{LOS NUEVOS HALLAZGOS. SISTEMÁTICA}

Orden ANSERIFORMES Bechstein 1804. Familia Anatidae Leach 1818. Género Tadorna Boie 1822. Tadorna sp. - tarro

Material: Extremo proximal de una ulna derecha (Amb, ph, STE-4, 23).

El tamaño del resto es superior al de Anas platyrhynchos. Se observa un olécranon más sobresaliente en Melanitta, Somateria y Mergus que en Tadorna, donde adquiere una forma muy redondeada. No se han encontrado diferencias entre los diseños morfológicos respectivos de T. tadorna y T.ferruginea en la porción de la ulna que se posee. Las dimensiones de ambas especies se solapan en la zona del rango de variación correspondiente a los valores más elevados (Woelfle, 1967). Las medidas del nuevo espécimen deAmbrona son grandes. Incluso la anchura diagonal proximal (DP) se situaría fuera del rango obtenido porWoelfle, si bien es cierto que no se deben generalizar los resultados del estudio de este autor porque la muestra de ejemplares de T. $f e$ rruginea con que trabaja es muy reducida. No se ha podido, en consecuencia, señalar la especie particular con las medidas del fragmento hallado (Tab. 1).

\begin{tabular}{|c|c|c|c|}
\hline & T. tadorna & T. ferruginea & Ambrona \\
\hline DP & $11.8-14.6$ & $13.7-14.6$ & 15.25 \\
\hline TP & $7.7-9.5$ & $9.0-9.8$ & 9.46 \\
\hline
\end{tabular}

Tab. 1. Medidas en centímetros de la ulna en Tadorna, según Woelfle (1967) (DP: medida desde el olécranon hasta el borde distal de la cotila externa. TP: medida desde el olécranon hasta el borde distal de la cotila interna).

\section{Género Anas Linnaeus 1758}

\section{Anas cf. acuta L. 1758 - ánade rabudo}

Material: Mitad craneal de un coracoides izquierdo (Amb, ph II, 43-E, 13). Sólo carece del ápice del procoracoides.
El coracoides es más robusto en Branta. Se observa que en Tadorna el tubérculo craneal presenta un mayor desarrollo. Es el tubérculo ventral el que, no obstante, posee mayor peso discriminante, dado que es de mayor talla en Anas que en Mergus, Netta y Aythya; siendo prácticamente indistinguible en Melanitta. En los esqueletos que se utilizaron para la identificación de este espécimen, la fosa del canal trióseo es menos profunda en $A$. acuta que en A. platyrhynchos; pero el coracoides es poco distintivo y el fósil es muy fragmentario para apoyar en él una determinación específica precisa. El hueso es de mayor talla que la que se ha observado en A. penelope (Woelfle, 1967). El ánade rabudo es un invernante común, aunque poco numeroso, en costas y aguas del interior (Coronado et alii, 1991).

\section{Anas sp. - ánade}

Material: Zona del manubrium del esternón (Amb, H 99/1 y H). Este resto procede de las extracciones que realizó C. Sesé.

Mediante la configuración de la espina dorsal del manubrium se distingue con facilidad Anas de Melanitta, Netta, Somateria, Mergus, Tadorna y Bucephala. En Anas, es pequeña; en los otros géneros, o es notablemente más amplia o ha llegado a escindirse en dos y a desaparecer. Atendiendo al tamaño del espécimen, éste no debió de corresponder a las formas más pequeñas del género Anas.

\section{Género Mergus Linnaeus 1758 \\ Mergus merganser L. 1758 - serreta grande}

Material: Extremo craneal de escápula derecha (Amb, I 100/2). También procede del material que obtuvo C. Sesé.

En Mergus, el acromion destaca más que en Anas y en Netta. Además, en Mergus se observa que el acromion se dirige más hacia el interior que en Somateria, en Melanitta y en Tadorna, de modo que en Mergus -también en Netta- el ángulo que forma esta prominencia con el eje central del hueso es muy agudo. La escápula alcanza en $M$. merganser una talla mayor que en $M$. serrator y $M$. albellus (Woelfle, 1967).

\section{Anseriformes indet.}

Material: Una diáfisis de húmero (Amb, ph, 47-G, 15) y una falange pedal (Amb, ph, 43-E, 6).

Húmero: La sección del fósil y el aspecto general que ofrece se corresponden con Anseriformes. La especie se hallaría entre las que poseen una talla superior a la de Tadorna. 
Falange: Posee el diseño propio del grupo de los anseriformes. Por sus dimensiones (longitud máxima: $18.16 \mathrm{~cm}$.; anchura horizontal máxima de la faceta proximal: $7.22 \mathrm{~cm}$.; anchura horizontal máxima de la faceta distal: $5.93 \mathrm{~cm}$.), esta falange se tendría que adscribir a un taxón de gran tamaño, similar a Anser.

\section{REGISTRO ORNÍTICO DEL CONJUNTO TORRALBA-AMBRONA}

Los yacimientos de Torralba y Ambrona están a una distancia de $3 \mathrm{~km}$. el uno del otro; con escasa diferencia entre sus respectivas cotas; cada uno, en una vertiente del valle que forma el río Masegar. Hasta muy recientemente, se consideraba que la diferencia cronológica entre ambas localidades, si la había, sería pequeña pues las dos se encontraban en la misma posición morfoestratigráfica y presentaban las mismas secuencias estratigráficas (Butzer, 1965; Howell et alii, 1995; Santonja y Villa, 1990). Como resultado de los últimos trabajos, se ha subrayado la diferente posición geomorfológica de estos dos yacimientos, lo que implicaría distintas edades (Pérez González et alii, 1997; e.p.). No obstante, los elementos avianos de ambas localidades parecen proceder de un mismo hábitat y haber vivido bajo similares condiciones climáticas, por lo que para tratar de esto pueden ser considerados conjuntamente.

La lista de aves que ofrecen Howell et alii (1995) puede constituir un principio de confusión. La han confeccionado sumando los taxones de Aguirre y Fuentes (1969), los de Sánchez (1990) y los de una versión antigua del presente trabajo. Además, sin consultar el material fósil, han hecho una corrección a las identificaciones de Aguirre y Fuentes (1969), a consecuencia de la cual aparece $c f$. Tetrao urogallus en lista de Howell et alii (1995) y no se incluye el Gallus cf. aesculapii de Aguirre y Fuentes (1969). Lo cierto es que el estudio de Sánchez (1990) se realizó sobre el mismo material que el de Aguirre y Fuentes (1969). Tampoco habría que admitir la paleoespecie $A n$ ser subanser, descrita por Jánossy (1983) (para una discusión, véase Sánchez, 1990). Por todo esto, la relación completa de las aves de Torralba y Ambrona constaría, en mi opinión, de los taxones identificados por Sánchez (1990), junto con los correspondientes a los nuevos restos del presente trabajo:

\section{Torralba:}

Tadorna ferruginea: fúrcula (E-9, 95, B-5, Q 2479).

Mergus serrator: escápula (H-6, 2, B-5, Q 2535).

Anatidae indet.: coracoides (F-9, 41, B-2C, Q 1379).

Porphyrio porphyrio: húmero (J-18, 44, B-2).

\section{Ambrona:}

Anser anser: radio (ph II, 43-D, 25), tarsometatarso (ph, 40-D, 15).

Tadorna ferruginea: ulna (ph, STE-12, 16).

Tadorna sp.: ulna (ph, STE-4, 23).

Anas acuta: coracoides (ph II, 43-E, 13).

Anas sp.: esternón (H 99/1 y H).

Mergus merganser: escápula (I 100/2).

Anseriformes indet.: húmero (ph, 47-G, 15), falange pedal (ph, 43-E, 6).

Fulica cf. atra: coracoides (ph II, 43-E, 92).

Vanellus vanellus: tibiotarso (47-E, 48).

\section{Localidad indeterminada:}

Anas strepera: coracoides (sin sigla).

La inclusión de los nuevos taxones en la lista de las aves de Torralba y Ambrona no modifica sustancialmente los rasgos paisajísticos y climáticos que se esbozaron en Sánchez (1990). Las especies identificadas son propias de terrenos pantanosos, encharcados y de masas de agua de escasa profundidad. Especies buceadoras, como las contenidas en Aythya y en la familia Podicipedidae están ausentes de este registro ornítico. No existe, pues, constancia de aguas profundas en la región.

Dos taxones aportados por este estudio, Anas cf. acuta y Mergus merganser, junto con $M$. serrator, alcanzan en la actualidad zonas europeas meridionales durante sus desplazamientos invernales. En España, la serreta grande es un visitante invernal raro, que frecuenta principalmente las costas o zonas adyacentes (Coronado et alii, 1991). Cuatro individuos de esta especie fueron observados por Bernis (1959) a comienzos de enero de 1959, en aguas interiores de la península ibérica, en el pantano El Rosarito, entre las provincias de Cáceres y Toledo.

Una implicación climática interesante a resaltar surge con la presencia de $P$. porphyrio, pues este animal, de hábitos sedentarios, es incompatible con temperaturas frías (Díaz et alii, 1996). El hallazgo en una asociación de fósiles de especies indicadoras de condiciones climáticas o ambientales muy diferentes entre sí ha conducido, en muchos traba- 
jos, a negar que se tratara de especies componentes de una misma biocenosis, aduciendo la posibilidad, bien de mezclas de niveles sedimentológicos adyacentes, bien de bajas tasas de sedimentación, que explicarían que restos de biocenosis muy distintas, pero sucesivas, aparecieran juntos. Subyacía una fidelidad estricta al principio del actualismo, en el sentido de que los conjuntos faunísticos del Cuaternario -casi 2 millones de años- han de encontrar un paralelo con los de nuestros días. Una explicación alternativa, sin suponer mezclas de niveles ni de biocenosis se dio en Sánchez (1996). Los restos que ahora se incorporan a los previamente conocidos insisten en la interpretación de unas condiciones climáticas no muy distintas de las actuales, con la salvedad de que quizá era más lluvioso, pues la zona de estos yacimientos estaba influenciada por áreas pantanosas o lagunas (Sánchez, 1990; 1996).

\section{BIBLIOGRAFÍA}

Aguirre, E. y Fuentes, C. (1969): "Los vertebrados fósiles de Torralba y Ambrona". Actas del VIII Congreso INQUA. En M. Ters (ed.): Études sur le Quaternaire dans le Monde. INQUA, París: 435-437.

Bernis, F. (1959): “Mergus merganser en el centro de España”. Ardeola, 5: 206-207.

Biberson, P. (1964): "Torralba et Ambrona. Notes sur deux stations acheuléenes de chasseurs d'éléphants de la Vielle Castille". Diputación Provincial de Barcelona, Monografías VI. Barcelona: 201-248.

Butzer, K.W. (1965): "Acheulean occupation sites at Torralba and Ambrona, Spain. Their geology". Science, 150: 1718-1722.

Coronado, R.; Del Portillo, F. y Sáez-Royuela, R. (1991): Guía de las anátidas en España. ICONA. Madrid.

Díaz, M.; Asensio, B. y Tellería, J.L. (1996): Aves ibéricas. I. No paseriformes. J.M. Reyero Ed. Madrid.
Howell, F.C. (1962): "El yacimiento achelense de Torralba (Soria)". Actas del VII Congreso de Arqueología (Barcelona, 1961): 110-116.

Howell, F.C.; Butzer, K.W.; Freeman, L.G. y Klein, R.G. (1995): "Observations on the Acheulean occupation site of Ambrona (Soria province, Spain)". Jahrbuch des Römisch Germanischen Zentralmuseum Mainz, 38: 33-81.

JÁNOSSY, D. (1983): “Die mittelpleistozän Vogelfauna von Presletice bei Prag (CSSR)". Schriftenreihe der geologische Wissenschaften, 19/20: 247-269.

Pérez González, A.; Santonja, M.; Gallardo, J.; AleixanDRE, T.; SesÉ, C.; Soto, E.; Mora, R. y Villa, P. (1997): "Los yacimientos pleistocenos de Torralba y Ambrona y sus relaciones con la evolución del polje de Conquezuela". Geogaceta, 21: 175-178.

Pérez González, A.; Santonja, M.; Mora, R.; Soto, E.; Sesé, C.; Ruiz Zapata, M.B.; Aleixandre, T.; Villa, P. y GALlARDo, J. (e.p.): "Investigaciones recientes (19901997) en los yacimientos achelenses de Ambrona y Torralba (Soria, España). Aproximación al contexto paleoambiental de fauna e industria en el complejo estratigráfico inferior de Ambrona". O Arqueologo Portugues.

SÁnCHEZ, A. (1990): "Aves de los yacimientos mesopleistocenos de Torralba y Ambrona (Soria, España)". Actas de las IV Jornadas de Paleontología. Salamanca: 349-357.

- (1996): "Aves fósiles del Pleistoceno ibérico: rasgos climáticos, ecológicos y zoogeográficos". Ardeola, 43 (2): 207-219.

Santonja, M. y Villa, P. (1990): "The Lower Paleolithic of Spain and Portugal". Journal of World Prehistory, 4 (1): 45-94.

SEsÉ, C. (1986): "Insectívoros, roedores y lagomorfos (Mammalia) del sitio de ocupación achelense deAmbrona (Soria, España)". Estudios geológicos, 42: 355-359.

WoELfLE, E. (1967): Vergleichend morphologische Untersuchungen an Einzelknochen des postcranialen Skelettes in Mitteleuropa vorkommender Enten, Halbgänse und Säger. Institut für Paläoanatomie, Domestikationsforschung und Geschichte derTiermedizin der Universität München. 